\title{
12
}

\section{The Interplay of Genetic and Cultural Factors in Ongoing Language Evolution}

\author{
Stephen C. Levinson and Dan Dediu
}

\begin{abstract}
This chapter discusses recent advances in our understanding of the complex interplay between cultural and biological factors in language change and evolution. Three "myths" (the independence of biological and cultural evolution, a fixed biological foundation for culture, and the cognitive uniformity of humans) are identified and falsified. Strong genetic biases are shown to affect language profoundly, using the example of village sign languages that emerge and complexify due to persistent high frequencies of genetic deafness in certain communities. Evidence is presented for the genetic bases of language and speech, and the extensive genetic variation within populations affecting them. Finally, it is proposed that in addition to intrapopulation variation, interpopulation differences in genetic biases that affect language and speech contribute to the emergence of linguistic diversity, through iterated cultural transmission across generations as well as communication and alignment within them. Thus, biological and cultural processes cannot be meaningfully separated when studying the cultural evolution of language.
\end{abstract}

\section{Cultural Evolution and Biological Evolution Are Two Sides of One Process}

This chapter is about the relationship between cultural and biological evolution, as evidenced in the domain of language. Many scholars with an interest in cultural evolution operate with a set of myths or fictions, tacitly holding something like the following:

1. Fiction of independence of biological and cultural evolution. Biological and cultural evolution are for practical purposes now independent processes, and despite the "curious parallels" between the diversification 
of species and of languages (as noted by Darwin 1871), the underlying principles are fundamentally different.

2. Fiction of a fixed biological platform for culture. Nevertheless, on a deep and ancient timescale, the two evolutionary tracks were in fruitful interplay and coevolved a now fixed platform for cultural liftoff: the big human brain, the language capacity, and the manual dexterity that made technology possible.

3. Fiction of the cognitive uniformity of the species. Since, generically, all humans have complex cognition, use language and make things with their hands, these capacities can be taken to be near uniform across the species: they form a constant background to cultural evolution.

In contrast, this chapter advances the following propositions:

1. Cultural and biological (genetic) evolution constitute twin tracks of an evolutionary process.

2. There are two-way feedback relations between the tracks.

3. These relations are ongoing.

4. There is significant variation within populations both with regard to genes and cultural variants, which supplies the "fuel" for evolutionary processes.

5. Even slight differences in the distribution of gene frequencies within populations can bias vertical and horizontal cultural processes and thus seed cultural evolution.

Propositions (1) and (2) have been much discussed. The general consensus is that, with a certain latitude over models, there is essentially no other way to explain the evolution of the capacities for culture (see, e.g., Boyd and Richerson 1985). Proposition (3) merely states Lyells's principle of Uniformitarianism: processes which used to operate on geologic timescales are still in operation now. It runs, though, directly into conflict with Fiction (2), the presumption that the biological platform for culture was achieved in prehistory and now remains essentially static. The fiction has currency because it is built into our cultural baggage, with humankind at the apex of the tree of life. In fact, of course, our rapid biological adaptation to cultural innovation is well attested (as in the development of adult lactose absorption in response to the culture of dairying, or the adaptation of the immune system to the diseases we have brought upon ourselves by migration, farming, behavior, sexual mores or misuse of antibiotics; Laland et al. 2010). Arguments that the different timescales of cultural and biological evolution sever the connection are unfounded; if one likens the twin tracks of culture and biology to obligate symbiosis (as between pollinators and flowering plants), it is obvious that differential rates of evolution are not an impediment to coevolution (Levinson 2006). Indeed, it is sobering to realize that the speed of biological change in our species is ever increasing (Hawks et al. 2007). 
Proposition (4) should be self-evident, but it runs against Fiction (3), which is the presupposition (or idealization) adopted at the birth of the cognitive sciences; namely that humans are, from a cognitive point of view, more or less clones, so that studying the minds or brains of, for example, highly educated Western undergraduates is to have sampled the entire variation (Henrich et al. 2010b; Levinson 2012a). However, it is Proposition (5) that has perhaps the most resonance for this volume, for it contains the following little time bomb: Cultural variation may not be wholly sui generis, for it may be seeded by small genetic differences across populations.

What this chapter explores, then, is the positive feedback relations between the cultural and biological tracks of evolution in the language domain, on the presumption that they continue to influence the direction of change.

\section{Village Sign Languages}

A compelling example of this mutual influence between biology and language is represented by village sign languages (Zeshan and de Vos 2012). Around the world, in developing nations without elaborate state institutions for handling special educational needs, population pockets can be found with a high incidence of congenital deafness. These are likely to be small village populations with considerable inbreeding, where the incidence of deafness may be tenfold higher than in the surrounding population (Winata et al. 1995; Scott et al. 1995). In these circumstances, sign language spontaneously arises, which over several generations begins to acquire the full expressivity we associate with spoken languages (De Vos 2012; Sandler et al. 2005). The hearing population also acquires the sign language, since relatives and friends are likely to be deaf. Deaf villagers thus become fully competent members of society, and marriage and reproduction takes place with little prejudice, so maintaining the high incidence of deafness in the village. Here in microcosm we see the interdependence of a cultural form of language, a specific sign language, and the genetic basis that perpetuates it. There is a positive feedback loop between cultural and genetic evolution.

In contrast, the major sign languages have arisen in the context of institutions for special education of the deaf. The exact history of their origins is often complex and little understood. French sign language is three hundred years old, but some are of recent origin. For example, Israeli sign language, ISL, arose during the formation of the state of Israel, when immigrants from different national institutional backgrounds and small-scale sign communities were brought together (Meir et al. 2010). The celebrated case of Nicaraguan sign language emergence is rather similar, where urban institutionalization of deaf children from different villages with some input from other national sign languages led to the rapid development of a new sign language (Senghas et al. 2005). The institutionalization of the deaf and the associated use of sign 
language and inter-deaf marriages represent another case of biology shaping culture, and, at least for some prevalent forms of genetic deafness, this cultural trait might have fed back on biology by increasing the frequency of the genes involved (Nance and Kearsey 2004; Arnos et al. 2008).

Village sign languages, however, are distinct (Meir et al. 2010): they have spontaneously arisen without input from another sign language wherever there is a high local incidence of deafness and no institutionalization of the deaf. The communities in which they are used may number a few thousand of whom up to $4 \%$ may be congenitally deaf (in the United States, the percentage in the general population is ca. $0.07 \%$ ). Nevertheless, up to two-thirds of the entire community may be sign language users, providing the critical mass of individual users necessary for language evolution to work (Senghas 2005). The village sign languages that have been researched seem to be of no great antiquity, with a depth of between $4-10$ generations, although there is also circumstantial evidence for recurrence in the same populations over greater time spans. There is enormous interest in what levels of linguistic complexity can arise on that kind of timescale (75-200 years) in such isolated settings without input from other signed languages (although, nota bene, with one-sided participation of hearing speakers of a spoken language). ${ }^{1}$

Here we focus on two of the better studied such languages. Al-Sayyid Bedouin Sign Language (ABSL) has arisen in a community of 3,500 with ca. 130 deaf adults over a period of ca. 75 years (Sandler et al. 2005). ABSL does not show any clear evidence of Hockett's "double articulation" (i.e., the recurrent use of meaningless elements like phonemes to construct higher-level units like words) and in that sense lacks a "phonology." Words themselves tend to have multiple conventional variants, with over a quarter having more than three acceptable alternate forms.

None of the village sign languages investigated so far shows any inflectional morphology, unlike the established national sign languages. However ABSL does employ noun compounding to modify nouns. Syntactic structures are simple, and despite having established an SOV (subject-object-verb) word order, clauses with two or more nominals are avoided in favor of a string of simple subject-plus-verb structures (so "GIRL STAND; MAN BALL THROW; GIRL CATCH" for "The man throws the ball to the girl"). As in other village sign language systems, there is no evidence for syntactic subordination, thus no evidence for the recursion or Merge expected by nativist generative grammarians.

Less grammatical work has been done on Kata Kalok (KK), a village sign language used for at least seven generations in Bengkala, northern Bali (Winata

1 There is, however, no significant structural borrowing from the spoken to the signed languages (e.g., word orders are distinct, vocabularies differently structured, syntactic and semantic calquing absent). Where there is "contamination," as it were, it is from the surrounding spoken gesture systems, which are likely to be incorporated and partially grammaticalized in the sign languages. 
et al. 1995; De Vos 2012). Again there is little evidence for double articulation or "phonology." Inflectional morphology is largely absent. Contrary to earlier reports, there appears to be no canonical word order, with avoidance of explicit multiple noun phrases, but when present all three orders (SVO, OVS, SOV) are equally likely. Nominals may function as modifiers and predicates, and order within phrases is very variable. Nominal semantics is more general than in the surrounding spoken language Balinese; for example KK has four color words, Balinese eleven, and KK has much less detailed kinship terminology.

Thus, village sign languages display simple structure, show much internal variation in allowable forms, do not show recursion, may not exhibit double articulation, and avoid multiple noun phrases that might cause confusion about "who did what to whom." Despite this, they have been put through the processes of recurrent learning by new generations and communication within generations. The evidence available so far from village sign languages is that it takes a great deal more than a half dozen generations to evolve a highly structured language. Experiments with iterated learning suggest that 6-10 generations might be required for highly structured artificial languages to evolve (Kirby et al. 2008), but these must be taken as very rough guides. Village sign systems are the only plausible cases of languages evolving de novo, since all spoken languages, as far as we know, descend from one or more other languages by direct descent or hybridization. They are also one of the few cases where we can readily detect a feedback from culture to biology (on top of the more general influence of biology on culture), although the general role of language skills in sexual selection and biological fitness is highly plausible.

From a genetic point of view, in both the KK and ABSL cases, a mutation was introduced in the population relatively recently (probably 7-8 generations ago for ABSL and 7-20 for KK) either from outside or through a spontaneous event in the population. Interestingly, these two mutations are different: for ABSL, the mutation affects the locus DFNB1 on chromosome 13q12, most probably the genes GJB2 and GJB6 (OMIM² 220290), while for KK the mutation disrupts the DFNB3 locus and more precisely the MYO15A gene on chromosome 17p11.2 affecting the hair cells in the cochlea essential for hearing (OMIM 602666) (Liang et al. 1998). However, both mutations, even if they affect different genes, result in a nonsyndromic hearing loss (i.e., deafness not accompanied by other problems or defects) starting before the onset of language acquisition (prelingual) in individuals carrying two copies of the mutation (homozygous). Individuals carrying a single copy of the mutation and a copy of the normal gene (heterozygous) have normal hearing, making the mutation recessive. Because both affected loci are on the nonsex chromosomes (autosomes) 13 and 17, respectively, a child will inherit a copy from its mother and one copy from its father. Thus, a child will be affected by hearing loss only

\footnotetext{
${ }^{2}$ Online Mendelian Inheritance in Man (OMIM), http://www.ncbi.nlm.nih.gov/omim, uses unique numeric identifiers for genetic disorders and genes.
} 
if the two copies inherited from both parents are mutated, meaning that both parents must be heterozygous carriers (with normal hearing) or homozygous deaf. In both communities, therefore, the probabilities of having a deaf child given the genetic structure of the parents are given in Table 12.1.

In both communities, there is a relatively high incidence of deafness due to homozygous individuals ( 3\% for ABSL and 2\% for KK), and such high frequencies of deafness have persisted for several generations due to high rates of inbreeding (within-community marriages). Inbreeding increases the chances that the two parents carry one (heterozygous) or two (homozygous) copies of the mutation, as they descend ultimately from the original carrier of the mutation in the population.

Such a situation in which congenital deafness persists could result in the long-term marginalization of the deaf. However, in both cases discussed here, not only are the deaf well integrated socially, including marriage and reproduction with other members of the wider community, but most hearing members of the communities are also able to communicate effectively with the deaf members. This has encouraged the feedback between the two systems: the culturally evolved sign language which allows deaf members to be fully fledged members of the society, and the inbreeding thus facilitated which maintains the strong strand of hereditary deafness. There has been some interesting mathematical and computational work that has addressed the population characteristics and assortative mating required to maintain this positive feedback relation between language and genes (Feldman and Aoki 1992; Nance and Kearsey 2004), but a more general model is needed.

We have focused on village sign languages as a microcosm to illustrate the interplay between genes and culture in the evolution of a language. Of course, the principles here are special, and the genetics of high-incidence deafness

Table 12.1 Probabilities (as percentages) of hearing and deaf children resulting from all possible marriages by parental genotype. $\mathrm{N}$ represents the normal allele, $\mathrm{M}$ the mutated, deafness-causing allele. Possible children genotypes are given by phenotypes; percent.

\begin{tabular}{|c|c|c|c|c|}
\hline & & Moth & er's genotype (phen & type) \\
\hline & & $\begin{array}{c}\text { NN } \\
\text { (hearing) }\end{array}$ & $\begin{array}{c}\text { NM } \\
\text { (hearing) }\end{array}$ & $\begin{array}{c}\text { MM } \\
\text { (deaf) }\end{array}$ \\
\hline & $\begin{array}{c}\mathrm{NN} \\
\text { (hearing) }\end{array}$ & NN (hearing; 100\%) & $\begin{array}{l}\text { NN (hearing; 50\%) } \\
\text { NM (hearing; 50\%) }\end{array}$ & NM (hearing; 100\%) \\
\hline 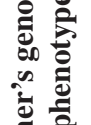 & $\begin{array}{c}\text { NM } \\
\text { (hearing) }\end{array}$ & $\begin{array}{l}\text { NN (hearing; 50\%) } \\
\text { NM (hearing; 50\%) }\end{array}$ & $\begin{array}{c}\text { NN (hearing; 25\%) } \\
\text { NM (hearing; 50\%) } \\
\text { MM (deaf; 25\%) }\end{array}$ & $\begin{array}{l}\text { NM (hearing; 50\%) } \\
\text { MM (deaf; 50\%) }\end{array}$ \\
\hline & $\begin{array}{l}\text { MM } \\
\text { (deaf) }\end{array}$ & NM (hearing; 100\%) & $\begin{array}{c}\text { NM (hearing; 50\%) } \\
M M \text { (deaf; 50\%) }\end{array}$ & MM (deaf; 100\%) \\
\hline
\end{tabular}


forces a modality change in language. Nevertheless, the examples serve as an important reminder of the power of genetic biases to send cultural evolution down novel paths. In the following, we will focus on much weaker effects, examining first the amount and nature of interindividual genetic variation affecting language and speech, and then looking into the effects of interpopulation differences on language change, diversity, and universal properties.

\section{The Fuel of Evolution: Genetic Variation in the Population as Evidenced in Language Performance}

We have mentioned the common assumption in the cognitive and social sciences that human evolution is somehow in abeyance, and that we all have the same essential cognitive endowments, linguistic abilities being a prime example: Fictions (2) and (3) above. In fact, linguistic abilities are clearly conditioned by genetic variation, and this variation can be found within both the clinical and normal population.

A first approach concerns the estimation of the heritability of aspects of speech and language. In essence, heritability is defined as the proportion of phenotypic variance explained by variance in the genotype, ${ }^{3} h^{2}=\operatorname{var}(G) / \operatorname{var}(P)$ (Visscher et al. 2008). Its estimation classically involves twin and adoption studies, but new methods of estimation from unrelated individuals have been recently developed. In twin studies, the phenotypes of monozygotic (identical; $\mathrm{MZ}$ ) and dizygotic (nonidentical; DZ) twins are compared; since MZ twins are genetically identical ${ }^{4}$ whereas DZ twins share on average $50 \%$ of their genes, the (narrow sense) heritability of the phenotype can be estimated. In its simplest form, ${ }^{5} h^{2}=2 \times\left(r_{M Z}-r_{D Z}\right)$, where $r_{M Z}$ and $r_{D Z}$ are the correlation between the phenotypes of the MZ twins and the correlation between the phenotypes of the DZ twins, respectively. Heritability can vary between 0 (genetic factors do not account at all for variation in the phenotype) and a maximum of 1 (all phenotypic variation is accounted for by genetic variation), and several caveats and limitations must be kept in mind when interpreting it (Visscher et al. 2008; Charney 2012).

There is a wealth of twin studies on various aspects of language (for a good review, see Stromswold 2001), and they suggest that most have a genetic component. For example, various disorders of speech and language usually show moderate to high heritabilities $\left(h^{2}>0.50\right)$, such as liability to stuttering, specific language impairment, or dyslexia. Likewise, an important genetic

3 There is a distinction between broad sense heritability (denoted $\mathrm{H}^{2}$ ) and narrow sense heritability $\left(h^{2}\right)$. Here we focus on the latter, given that it is easier to estimate and it is generally used in the literature. This is also the additive component of genetic variation upon which selection can work.

4 Ignoring de novo mutations.

5 Presently, more complex methods based on structural equation modeling are increasingly used. 
component was found for some aspects of normal variation in language and speech such as vocabulary size and second language learning. In addition, the physical aspects of producing speech are under genetic influence as shown by the heritability of the shape and size of the hard palate, tongue size and shape and of various acoustic properties of speech due to vocal tract anatomy and physiology. Heritability studies establish prima facie reasons to suspect genetic causal correlates of phenotypic differences, but they do not themselves pinpoint the genes or pathways involved, which need a different set of methods.

A very successful and productive research program was initiated by the discovery (Lai et al. 2001) that the FOXP2 gene is responsible for a complex pathology ("developmental verbal dyspraxia”) that affects speech and language (OMIN 602081). This gene is highly conserved across mammals and, against this background, the human form (which we share with our cousins, the Neandertals; Krause et al. 2007) has specific mutations that might be related to the emergence of speech and language in our lineage. It is currently uncertain whether polymorphisms ${ }^{6}$ in FOXP2 affect normal variation in speech and language, but there are early indications that they may, at least at the neural level (Pinel et al. 2012). FOXP2 gave us an entry point for exploring the genetic underpinnings of speech and language because it is a regulatory gene (Fisher and Scharff 2009). The identification of other genes regulated by FOXP2 proves to be an active research field, leading to the discovery of genes such as CNTNAP2 (Vernes et al. 2008) which is also involved in language pathologies.

Another syndrome that has offered valuable insights is dyslexia (Scerri and Schulte-Körne 2010), a spectrum disorder which manifests as a reading disability but which often seems nevertheless to reflect deeper phonological and linguistic processing problems. Risk factors for dyslexia are association with alleles of the genes KIAA0319 and ROBO1 among others. Interestingly, it was recently found that $R O B O 1$ is involved in normal variation in Non-Word Repetition (Bates et al. 2011), a task thought to measure a component essential for language acquisition. In this sort of way, research into syndromes can reveal variants that have milder effects in the general population.

To summarize, during the last decades we have accumulated a lot of evidence that (a) most normal and pathological aspects of speech and language have a genetic component, (b) these genetic foundations are extremely complex (Fisher 2006), and (c) there is extensive variation between individuals. This last point is fundamental for understanding the relationships between language and genes and needs to be emphasized: close investigation reveals variation between normal individuals in almost all aspects of speech and language one cares to consider. This includes variation in grammaticality judgments (e.g., Schütze 1996; Dąbrowska 1997) and sentence processing (Farmer et al. 2012), suggesting that the normal, fully competent native speaker's language carries

6 That is, normal variants of the gene, as opposed to the catastrophic mutations, which give rise to developmental verbal dyspraxia. 
that individual's genome signature in subtle ways. It must be highlighted that this signature is not direct and deterministic, but modulated by the environment, such that the individual differences result from the complex interaction of the genetic background and the individual's experiences (Lieven, this volume). Such a signature may remain hardly discernible until the individual's language system is under stress or when the cultural and social circumstances change, such as when literacy became a widespread and essential requirement, unmasking hitherto hidden genetic variation in the form of what we now diagnose as dyslexia (see Tomblin and Christiansen 2009). In short, just as we are short or tall, fat or thin, good at running or dexterous with our hands, so all the myriad capabilities that underlie language capacity vary within any population.

We turn now briefly to the differences across populations. Cavalli-Sforza et al. (1994) produced a magnum opus that estimated the most likely phylogenetic trees for worldwide human groups based on blood groups and other classical genetic markers, and found close matches to the trees so obtained with the boldest suggestions for the phylogenetics of the world's language families then available. It is now conceded that both kinds of trees were flawed (e.g., SimsWilliams 1998). They presumed successive splitting with minimal hybridization, which is wrong for both language and genes, and used language trees that few linguists now subscribe to. Since then, with the full sequencing of the genome, the simplicity of the phylogenetic story has unraveled, and the many factors from disease to ecology shaping genetic diversity have become apparent (Jobling et al. 2004; Novembre et al. 2008). Even small gene trickle between Pacific populations, for example, has wiped out close correlations with language families in that area. Spectacularly, recovery of archaic DNA shows that some populations have inherited genes from different premodern humans, with out-of-Africa peoples picking up Neandertal genes (Green et al. 2010), and Australians and Papuans further picking up genes from the Denisovans (Reich et al. 2011), a sister branch to the Neandertals. Still, when the dust settles from the intensive work on the human genome now in progress, we can be fairly sure that the migration history and kinship of most human groups will be at least partially recoverable from the genetics (see Novembre et al. 2008; Paschou et al. 2010).

Human groups then tend to have relatively different gene pools, within which the gene variants governing language-related phenotypes will no doubt be shown in due course to vary in kind or more likely proportion. It is, however, important to remember that this kind of variation across human groups is a minor part of the story. Modern humans are genetically a very homogeneous species when compared to other mammals (Barbujani and Colonna 2010), due to our evolutionary history involving small population sizes and repeated bottlenecks, with few differences between populations due to selection, such as in the immune system (Mukherjee et al. 2009) or skin color (Jablonski and Chaplin 2010). This is reflected in the distribution of genetic differences: about $85-90 \%$ of the genetic variation is between the individuals of the same 
population with only 10-15\% lying between populations, and even these form smooth clines without sharp boundaries that could be taken as anything like "human races" (Cavalli-Sforza et al. 1994). These small differences between populations, though distinctive, are mostly due to differences in the frequency of ubiquitous (“cosmopolitan”) genetic variants and very rarely to group-specific ("private”) ones.

To summarize this section:

- We are beginning to discover the genes that build the brain and the vocal tract and make language and speech possible.

- These discoveries rest on significant variation in genes within populations; these govern distinctive phenotypes, which provide a reservoir to be exploited for language evolution.

- There is also lesser but significant variation in gene pools across populations in different parts of the world.

\section{Language Diversity Seeded by Genetic Variation}

We wish to put forward a startling hypothesis: Genes may be partly responsible for the cultural diversity of language, and it certainly is the antithesis of Fiction (1). They may play this role in two ways:

1. Genetic diversity within populations harbors a range of potentially culturally reinforcable language phenotypes.

2. Small genetic differences between populations may slightly bias cultural transmission and so tip cultural evolution in particular directions.

We should emphasize right away that this is not a theory of genetic determinism of culture, nor a kind of closet racism. The spectrum of phenotypic possibilities in Hypothesis (1) will be mostly shared across populations right around the world. The biases in Hypothesis (2) just amplify tiny imbalances by cultural processes and are highly contingent on many other factors, just as in chaos theory where small differences in input conditions can lead to wildly different outcomes.

Let us begin with Hypothesis (1), which is largely speculative but plausible. As noted, most human genetic variation is shared across widely spread populations; just as there are tall versus short, lighter- versus darker-skinned, thin versus fatter individuals in every population, so there are potential lispers, stammerers, hyper-multilinguals, gifted poets, singers, or public speakers in every population. In addition, as remarked earlier, the variable genetic substrates may often be masked by adequate linguistic performance. Think of a language as a mapping from sound (or sign) to meaning: there may be multiple possible algorithms that will do the mapping, and there may be multiple ways that any one of those algorithms can be instantiated in wetware. Given the fundamental 
variation in language types around the world (Evans and Levinson 2009a; see also Evans, this volume), it is likely that different types of language favor different algorithms. Consider, for example, that some languages have contrastive tone on words and some do not. Chinese speakers and even speakers of tonal dialects of European languages show greater activation of right hemisphere neural circuitry, probably because the right hemisphere better handles the 200 ms processing window required. Yet we already know that speakers of nontonal languages show considerable variation in the degree of right hemisphere language processing, that this is reflected in cortical structures and linked to biological factors like sex (Catani et al. 2007). In this way there is a reservoir of potentiality in any population: some individuals will be preadapted for more efficient processing of a tonal language, others for a nontonal language. A particular cultural form of language exploits the special facility of some individuals and makes the rest work harder. ${ }^{7}$

In a similar way, it is likely that languages with surface phrase structure and fixed order must be processed differently than those with case markers and scrambled word order; the former predisposes a more syntax-driven route to parsing, the latter a more semantic (thematic-role) driven route. Shallow, "good enough" processing shows we all probably use both routes, but the differences in grammaticality judgments mentioned earlier probably reflect the graded interindividual differences in the use of various strategies. At present we know little about intrapopulation differences in language processing due to the unfortunate Fiction (3) of cognitive uniformity, which has dominated the cognitive sciences (Levinson 2012a). Variation is avoided by using Western undergraduate participants for experiments (Henrich et al. 2010b)_but even in this very restricted population, variation, of course, exists (Farmer et al. 2012) — and by subsuming variation in group averages, while brain imaging has historically mapped results onto a generalized brain and swept the lefthanders and even the "other" sex under the rug.

Let us turn now to Hypothesis (2): genetic differences between populations may slightly bias cultural transmission and intragenerational communication and so tip cultural evolution in particular directions. Hypothesis (1) is the idea that a single population encompasses most of the variation. Hypothesis (2) is based on the assumption that the variation is not equally distributed across all populations, and that this can have consequences for the likely direction of cultural evolution. It is clearly in line with what we know about the distribution of genetic diversity in general across the human species, as discussed above.

7 An application of the same idea to nonlinguistic material may perhaps be found in observations about cultural "techniques du corps" made by Mauss. Some traditional populations throw overhand, others underhand; some swim breaststroke, others prefer crawl; some relax by squatting on their haunches while others stand on one leg, and so forth. Big calf muscles make it hard to squat, thin calves make it easy, and in this way genetic variation may seed cultural norms (Mauss 1973). 
Hypothesis (2) has some empirical backing. First, computational and mathematical modeling of language transmission shows that weak biases in individual processing get amplified through cultural transmission, resulting in universal tendencies when the bias is shared by all individuals (Kirby et al. 2007). Experimental studies of iterated learning provide more evidence that the transmission of language across chains of human participants amplifies much weaker biases, such as a bias towards systematicity (Smith and Wonnacott 2010). Further modeling shows that when the bias is unequally distributed across populations, different structural solutions recurrently emerge (Dediu 2008). In short, innate biases in the form of slightly different proportions of alleles in a population are sufficient to breed extensive cultural differences.

Slight differences in the facility with which sounds can be produced would constitute one of these kinds of biases. Consider that the authors of this paper both have their linguistic Achilles' heels: one had a childhood lisp and the other cannot do his alveolar trills to this day, but of course not all languages have sibilants or trills, and thus we could both have won elocution prizes in the appropriate cultures. A population heavily seeded with Levinsons and Dedius would not have evolved a language with sibilants and trills. There is some empirical evidence for the effects of these production biases on linguistic typology. Naturally enough, vocal tract anatomy exhibits structured variation across the populations of the world. It has been noted that some of these differences likely contribute to the probabilities of certain phoneme inventories. For example, Ladefoged (1984) noted differences in the second formant (F2) between the otherwise similar vowel systems of Yoruba and Italian, arguing that it might be due to differences in the anatomy of the upper tract between the typical speakers of these languages, ideas that have been further examined by Dediu (2011b) and Ladd et al. (2008). Another example concerns the shape and dimensions of the hard palate (the bony roof of the mouth), which is known to vary across populations in the degree to which it is wide and flat, or narrow and domed (Sugie et al. 1993; Byers et al. 1997), and known to be under genetic influences (Townsend et al. 1990; Dellavia et al. 2007). These differences induce differences in tongue contact, and thus have acoustic correlates. It is probable that a high-domed palate facilitates the production of apical retroflex consonants (sounds produced with the tip of the tongue curled back, such as [t] and [d]), even though anyone can learn to produce these sounds. The hypothesis advanced here is that there will be a positive correlation between the proportions of palate shapes in a population and the phoneme inventories likely to arise. This is because palate shape would constitute one of these weak but insistent biases that have been shown in modeling to induce populationlevel cultural differences.

Probably the best-supported proposal to date for such a genetic bias channeling cultural evolution links the distribution of linguistic tone (the use of voice pitch to convey lexical and grammatical distinctions; Yip 2002) and the distribution of alleles of two genes involved in brain growth and development, 
ASPM and Microcephalin (Dediu and Ladd 2007). The frequency of certain variants of ASPM and Microcephalin is correlated with the presence or absence of linguistic tone in the language used by those populations, and this correlation survives multiple controls such as shared history and language and genetic contact. It is not entirely clear what the underlying mechanism is which constitutes the learning or production bias, but a recent report (Wong et al. 2012) suggests both behavioral and neurophysiological correlates of ASPM variants in pitch processing. Thus the strong suggestion is that the odds of developing a tone language by cultural evolution are slightly weighted by the proportion of these alleles in a population. ${ }^{8}$

To summarize, then, there is both plausibility and prima facie evidence that population genetics provides both a reservoir that would facilitate the huge range of alternate cultural possibilities open to the species and, at the same time, biases the local outcomes of cultural evolution through structured variation in the local population.

\section{Conclusions}

Polite fictions of the current independence of cultural and biological evolution, of biological evolution being now in abeyance and the cognitive uniformity of the species, need to be swept away. Instead, our theoretical and empirical investigations into cultural evolution need to be informed by the feedback relations between biological and cultural evolution (see also Lieven, this volume). The example of village sign languages serves as a startling reminder that this feedback is crucial for understanding ongoing cultural evolution.

We emphasize the pervasiveness of genetic variation in any population and its inevitable links to phenotypic (cognitive or behavioral) variation. We outlined the rapidly developing insights into genetic factors in language capacities, and how a single population harbors most of the variation in the species, but nevertheless local populations evidence distinct proportions of genetic variants. These slight local population differences may be sufficient to seed cultural differences and there is some evidence that these processes actually occur in the language domain.

The crucial message for this volume is that we cannot sensibly divorce cultural evolution from biological evolution. The two are intertwined. The distribution of genetic variants can bias the direction of cultural evolution, and cultural evolution can, in principle, channel biological evolution. Theories like twin-track models of coevolution of culture and biology, or the concept of niche construction (Odling-Smee et al. 2003), should always be borne in mind.

8 Of course, it is entirely possible that the reverse process, whereby speaking a tone language generates weak selective pressures on the processing and acquisition of specific cues, is active as well, but this probably cannot explain the observed genetic differences. 
Language is par excellence a bio-cultural hybrid: a cultural system that runs on biological infrastructure and offers general insights into cultural evolution.

\section{Acknowledgments}

We wish to thank the participants of the Forum for extensive discussions and, in particular, Simon Garrod for highlighting the importance of horizontal processes in amplifying weak genetic biases. We also thank one anonymous reviewer for constructive comments and suggestions. 